\title{
To Prognosticate the Final Outcome in a Traumatic Brain Injury Patient based on their Electrolyte Changes
}

\author{
Dheeraj Kumar Raj ${ }^{1}$, Pankaj Arora ${ }^{2}$, J.P.Sharma ${ }^{3}$, Puneet Malik², Amulya Aggarwal ${ }^{1}$ \\ ${ }^{1}$ Post Graduate Residents, Department of General Surgery, ${ }^{2}$ Assistant Professor, Department of Neuro Surgery, \\ ${ }^{3}$ Professor, Department of General Surgery, Shri Guru Ram Rai Institute Of Medical And Health Sciences,
} Dehradun, Uttarakhand.

\section{Introduction}

Dyselectrolytemia is very common in head injuries patients and it is likely due to abnormality in serum sodium, potassium, calcium, phosphate, chloride. Serum Sodium is the most common and important electrolyte abnormality among these electrolytes. Proper management of dyselectrolytemia in such patient following a head injury is most important . Proper in time detection followed by appropriate treatment not only improves neurological status but also decrease morbidity and mortality. Aims \& Objective : To prognosticate the final out come in a traumatic brain injury patient based on there electrolyte changes. Material \& Method : A Hospital based Prospective type Cross Sectional Study . The study was conducted over a period of 18 month In our institute. This study includes all Traumatic Brain Injury patients without poly trauma who were admitted through Emergency as well as OPD. This study exclude patients with any co morbidity like diabetes mellitus, hypertension, thyroid derangement and poly trauma etc. Observation \& Results : We observed significant difference of sodium, potassium, and chloride levels following TBI recorded at the time of admission, 24 hours, after 4 days, 8 days, 12 days and 16 days. There was positive correlation of hyponatremia, hypokalemia, hypochloremia, with outcome of the patient. Conclusion : we concluded that electrolyte imbalance following traumatic head injury is an important cause to look for in patient monitoring. Sodium potassium and chloride chief electrolyte of concern. Serum calcium and Phosphate levels also under goes notable changes.

Keywords: Traumatic brain injury, serum electrolyte derangements.

\section{Introduction}

Traumatic brain injuries (TBIs) is a leading cause of morbidity, mortality, disability and socio economic losses in India and in other developing countries. In India, over $1,00,000$ people die due to road traffic accidents each year ${ }^{(1)}$ and nearly $50-60 \%$ of them are hospitalized for brain injury. ${ }^{(2)}$ Electrolyte derangements are common sequelae of traumatic brain injury. Dyselectrolytemia is very common in head injuries patients and it is likely due to abnormality in serum sodium, potassium, calcium, phosphate. It may be due to use of intravenous fluids,

\section{Corresponding author :}

Dr. Pankaj Arora

E-mail: parorafzr@yahoo.com diuretics, syndrome of inappropriate $\mathrm{ADH}$ secretion and cerebral salt wasting. Serum Sodium is the most common and important electrolyte abnormality among these electrolytes, both hyponatremia and hypernatremia can occur. More so changes in potassium chiefly hypokalemia ${ }^{(3)}$ and fluid content ${ }^{(4)}$ are also encountered in clinical practice. ${ }^{(5)}$ There are some different causes and among them most common being syndrome of inappropriate anti-diuretic hormone secretion (SIADH) ${ }^{(6)}$, Cerebral salt wasting $(\mathrm{CSW})^{(5)}$ use of diuretics like Furosemide and Mannitol. ${ }^{(4)}$ Age is another important factor that also greatly affects morbidity and mortality. Advancing age has poor outcome. ${ }^{(8)}$ However, patients may deteriorate after initial improvement even after a week due to electrolyte disturbances chiefly sodium. (11) So proper management of dyselectrolytemia in such 
patient following a head injury is most important. ${ }^{(9)}$ Apart from Sodium and Potassium, chloride is also is important electrolyte abnormality associated with a variety of clinical manifestations in patients with traumatic brain injury. ${ }^{(12)}$ Initially from the development of tetany ${ }^{(13)}$ to seizures all can happen following derangement in serum calcium. Abnormal responses of neurons to stimulation secondary to accumulation of intracellular calcium in traumatic brain injury are responsible for these features. ${ }^{(14)}$ Abnormality in serum phosphate following a traumatic brain injury is also been observed. Serum phosphate is also a major intracellular anion and plays an important role in many biochemical pathways relating to normal physiologic functions, especially in maintaining muscle tone. ${ }^{(15,16)}$ As Hypophosphatemia has been shown to be associated with muscle weakness, including weakness of respiratory muscles, and with respiratory infection. Proper in time detection followed by appropriate treatment not only improves neurological status but also decrease morbidity and mortality. ${ }^{(16)}$

\section{Material \& Method}

A Hospital based Prospective type Cross Sectional Study. The study conducted over a period of 18 month.
This study includes all Traumatic Brain Injury patients without poly trauma who were admitted through Emergency as well as OPD will be included in the study. This study exclude patients with any co morbidity like diabetes mellitus, hypertension, thyroid derangement etc.

The severity of TBI according to GCS score [with in $48 \mathrm{hr}]$ is as follow:

- $\quad$ Severe $\mathrm{TBI}=3-8$

- $\quad$ Moderate TBI=9-12

- $\quad$ Mild TBI=13-15

The severity of head injury were assessed by Glasgow coma scale [GCS] and type of injury were assessed by head computed tomography [CT]. Serum electrolytes [serum sodium, potassium, calcium ,Chloride and phosphate], GCS, ESR, CRP were measured at time of admission in emergency before starting intravenous fluid and repeated at 24 hours, $4^{\text {th }}, 8^{\text {th }}, 12^{\text {th }}, 16^{\text {th }}$ days after resuscitation.

All patient received standard treatment as per institutional protocol for Traumatic Brain Injury.

The normal value taken in to the consideration in this study are as follow -

\begin{tabular}{|l|l|}
\hline Electrolyte & Normal Value [range] \\
\hline S. Sodium & $135-145 \mathrm{mEq} / \mathrm{l}$ \\
\hline S. Potassium & $3.5-5.5 \mathrm{mEq} / \mathrm{l}$ \\
\hline S. Calcium & $8.6-10.3 \mathrm{mg} / \mathrm{dl}$ \\
\hline S. Phosphorous & $2.5-4.5 \mathrm{mg} / \mathrm{dl}$ \\
\hline S. Chloride & $96-106 \mathrm{mmol} / 1$ \\
\hline C Reactive Protein & $0-3 \mathrm{mg} / \mathrm{l}$ \\
\hline ESR & $0-20 \mathrm{~mm} / \mathrm{hr}$ \\
\hline
\end{tabular}

Statistical data analysis - In order to establish correlation between electrolytes and outcome in the present study, we used chi square test. 
Findings

DISTRIBUTION TABLE 1 CORRELATION OF PATIENT OUTCOME WITH SODIUM

\begin{tabular}{|l|c|c|c|c|}
\hline Electrolyte & Improved & Expired & Deteriorated & Lama \\
\hline Hypernatremia [60] (30\%) & 23 & 24 & 10 & 3 \\
\hline Normal sodium[114](43\%) & 95 & 14 & 4 & 0 \\
\hline Hyponatremia [26](13\%) & 22 & 2 & 2 & 4 \\
\hline Total [200] & 140 & 40 & 16 & 0 \\
\hline
\end{tabular}

Hyponatremia is present in 26(13\%) patients, hypernatremia present in 60(30\%) patients and normal sodium level present in $114(43.5 \%)$ patients. We found that hypernatremia is more related to poor outcome (i.e) of the patient. $[p=<0.05]$

TABLE 2 CORRELATION OF PATIENT OUTCOME WITH POTASSIUM

\begin{tabular}{|l|c|c|c|c|}
\hline Electrolyte & Imporoved & Expired & Deteriorate & LAMA \\
\hline Hypokalemia [55] & 18 & 25 & 12 & 0 \\
\hline Hyperkalemia [21] & 21 & 3 & 1 & 4 \\
\hline Normal potassium[120] & 101 & 12 & 3 & 4 \\
\hline Total patients [200] & 140 & 40 & 16 & 4 \\
\hline
\end{tabular}

Hypokalemia present in 55(27.5\%) patients, hyperkalemia present in 25(12.5\%) patient and normal potassium level present in $120(60 \%)$ patients. hypokalemia is more related to poor out come of patients. . [ $p=<0.05$ ]

TABLE 3 CORRELATION OF PATIENT OUTCOME WITH CALCIUM

\begin{tabular}{|l|c|c|c|c|}
\hline Electrolyte & Improved & Expired & Deteriorate & LAMA \\
\hline Hypocalcemia [50] & 24 & 25 & 1 & 0 \\
\hline Hypercalcemia [15] & 14 & 0 & 1 & 4 \\
\hline Normal calcium [135] & 102 & 15 & 14 & 4 \\
\hline Total [200] & 140 & 40 & 16 & 4 \\
\hline
\end{tabular}

50(25\%) patients has hypocalcemia, 15(12.5\%) patient has hypercalcemia and rest 135(67.5\%) patients has normal calcium level. We observed that Hypocalcemia is more related to poor outcome of the patient. . [ $p=<0.05]$ 
TABLE 4 CORRELATION OF PATIENT OUTCOME WITH PHOSPHATE

\begin{tabular}{|l|l|l|l|l|}
\hline Electrolyte & Imporoved & Expired & Deteriorate & LAMA \\
\hline Hypophosphatemia [70] & 45 & 20 & 4 & 1 \\
\hline Hyperphosphatemia [20] & 8 & 5 & 6 & 1 \\
\hline Normal phosphate [110] & 87 & 15 & 6 & 2 \\
\hline Total [200] & 140 & 40 & 16 & 4 \\
\hline
\end{tabular}

Hypo-phosphatemia present in 70(35\%) patients, hyper-phosphatemia present in $20(10 \%)$ patient and normal potassium level present in 110(55\%) patients. very little role of this electrolyte in the outcome of patient. . [ $p=<0.05$ ]

TABLE 5 CORRELATION OF PATIENT OUTCOME WITH CHLORIDE

\begin{tabular}{|l|c|c|c|c|}
\hline Electrolyte & Improved & Expired & Deteriorate & LAMA \\
\hline Hypochloremia [16] & 10 & 5 & 1 & 0 \\
\hline Hyperchloremia [54] & 24 & 28 & 1 & 1 \\
\hline Normal chloride [130] & 106 & 7 & 14 & 3 \\
\hline Total [200] & 140 & 40 & 16 & 4 \\
\hline
\end{tabular}

67(28\%) patients has Hypochloremia, 12(5\%) patient has hyperchloremia and rest 161(67\%) patients has normal chloride level, hyperchloremia more related to poor out come of patients. [ $p=<0.05]$

TABLE 6: CORRELATION OF PATIENT OUTCOME WITH CRP LEVELS

\begin{tabular}{|l|l|l|l|l|}
\hline Electrolyte & Imporoved & Expired & Deteriorate & LAMA \\
\hline Low CRP & -- & -- & -- & - \\
\hline High CRP [170] & 116 & 35 & 15 & 4 \\
\hline Normal CRP [30] & 24 & 5 & 1 & 0 \\
\hline Total [200] & 140 & 40 & 16 & 4 \\
\hline
\end{tabular}

Patients have raise level of C-reactive protein are 170 in number,25 patients has normal level CRP. [ $p=<0.05]$

\section{Discussion}

We measured serum electrolytes such as serum sodium, potassium, calcium, chloride and phosphate ESR, CRP and GCS at time of admission in emergency before starting intravenous fluid and repeated at 24 hours, 4th, 8th, 12th, 16th days after resuscitation.

SODIUM -In present study out of 200 patients. Hyponatremia is present in $26[13 \%]$ patients, 
hypernatremia present in $60[30 \%]$ patients and normal sodium level present in 114[43.5\%] patients.

- Hyponatremia present in $26[13 \%]$ patients, out of these 22 were improved, 2 patients deteriorates and 2 patients are expired.

- Hypernatremia present in $60[30 \%]$ patients, out of these 23 patients were improved, 10 patients deteriorated and 24 patients were expired.

- Normal sodium level seen in 114 [77\%] patients, out of these 95 were improved, 4 patients were deteriorated and 14 patients were expired.

We found that hypernatremia is more related to poor outcome of the patients.

Elhassan et al studied 210 patients of TBI in this study, Authors found that there was an association between hypernatremia and poor outcome after TBI. ${ }^{(19)}$

POTASSIUM - out of 200 patients, hypokalemia present in 55[27.5\%] patients, hyperkalemia present in $25[12.5 \%]$ patient and normal potassium level present in $120[60 \%]$ patients.

- Hypokalemia is present in 55 patients, out of these 18 patients were improved, 25 patients expired and 12 patients deteriorated.

- Hyperkalemia present in 25 patient out of which 21 patients were improved, 3 patients were expired, 1 patient were deteriorated .

- Normal potassium level was present in 120 patients, out of these 101 patients were improved, 12 patients were expired and 3 patients were deteriorated and 4 gone LAMA.

- We concluded that hypokalemia is more related to poor out come of patients.

Suman et al found that \%], Hypokalemia [21.58\%], Hyperkalemia [17.77\%], they concluded that Hypokalemia is second most electrolyte abnormality after Hypernatremia for poor outcome of patient. ${ }^{(17)}$

CALCIUM -in present study out of 200 patient with traumatic brain injury, 50[25\%] patients has hypocalcemia, 15 [12.5\%] patient has hypercalcemia and rest $135[67.5 \%]$ patients has normal calcium level.
- Hypocalcemia present in 50 patients out of these 24 patients were improved, 25 patients expired and 1 patients deteriorated.

- Hypercalcemia present in 15 patients, out of which 14 patients were improved, and 1 patient was deteriorated.

- Normal calcium level seen in 135 patients, out of these 102 patients were improved, 15 were expired, 14 patients were deteriorated and 4 patients went LAMA.

We observed that Hypocalcemia is more related to poor outcome of the patient.

Gupta et al compared all electrolyte values in two groups taking head injury patient and concluded that Calcium levels under goes minute notable changes. ${ }^{(20)}$

Mirza et al [2013] studied the calcium level in TBI and concluded that serum calcium levels show little derangements. ${ }^{(18)}$

Suman et al obseved in there study that hypocalcaemia present in $11.4 \%$ of total patients studied which was more related to poor outcome. ${ }^{(17)}$

PHOSPHOROUS - out of 200 patients, hypophosphatemia present in $70[35 \%]$ patients, hyperphosphatemia present in $20[10 \%]$ patient and normal potassium level present in $110[55 \%]$ patients.

- Hypophosphatemia - present in 70 patients out of which 45 patients were improved, 20 patients were expired, 4 patients were deteriorated and 1 patient were gone LAMA.

- Hyperphosphatemia - present in 20 [10\%] patients out of these 8 patients were improved, 5 patients expired and 6 patients deteriorates 1 patient were gone LAMA.

- Normal phosphorous level seen in 110 patients, out of these 87 patients were improved, 15 expired and 6 patients were deteriorates 2 patients were gone LAMA.

Our study conclude that there is very little role of this electrolyte in the outcome of patient, hypophosphatemia more related to poor outcome. 
Suman et al. They concluded that there is no significant role of this electrolyte in the outcome of patients. ${ }^{(17)}$

CHLORIDE - In present study out of 240 patient with traumatic brain injury, 67[28\%] patients has hypochloremia, 12 [5\%] patient has hyperchloremia and rest $161[67 \%]$ patients has normal chloride level.

- Hypochloremia present in 16 [8\%] patients out of these 10 patients were improved, 5 patients expired and 1 patient deteriorated.

- Hyperchloremia present in 54 patients out of these 24 patients were improved, 28 patients expired and 1 patients deteriorated 1 patient gone LAMA.

- Normal chloride seen in 130 patients, out of these 106 patients were improved, 7 were expired and 14 patients were deteriorated, 3 patients gone LAMA.

This study concluded that hyperchloremia more related to poor outcome of patients.

C REACTIVE PROTEIN - in the present study 200 patients of traumatic brain injury included. Patients have raise level of C-reactive protein are 170 in number, 25 patients has normal level CRP.

High CRP seen in 170 patients, out of these, 116 were improved, 35 were expired and 15 were deteriorated 4 patients gone LAMA.

Normal CRP seen in 30 patients, out of these 24 patients were improved, 5 patients expired and 1 patient was deteriorated.

Our study concluded that raised level of C-reactive protein more related to poor outcome of patients.

Naghibi et al In the female group, raised CRP level was positively correlated with the length of ICU stay and the duration of mechanical ventilation. ${ }^{(23)}$

Zolin et al found that high CRP level was significantly correlated with the length of ICU stay and the duration of mechanical ventilation. ${ }^{(24)}$

ESR--in the present study include 200 patients of traumatic brain injury .165 patients have raise level of ESR and 35 patients has normal level.
ESR -

High ESR seen in 165 patients, out of these, 111 were improved, 36 were expired and 14 were deteriorated 2 patients gone LAMA.

Normal ESR seen in 35 patients, out of these 29 patients were improved, 4 patients expired and 2 patient was deteriorated 2 patients gone LAMA.

Our study concluded that raised level of ESR more related to poor out come of patients.

\section{Conclusion}

We suggests that electrolyte imbalance following traumatic head injury is an important cause to look for in patient monitoring.

Sodium, Pottasium and Chloride are the chief electrolyte of concern.

Serum calcium and Phosphate levels under goes minute changes. Based on CT scan findings several traumatic brain injuries associated with various electrolytes derangements are of important concern especially with in first 24 hours after resuscitation.

We recommend serum Sodium, potassium and chloride along with CRP levels should be included in the work up of the patient as a predictor of prognosis and should be monitored and corrected from time to time.

\section{Conflict of Interest- NIL}

\section{Source of Funding- NIL}

Ethical Clearance- Institutional ethical committee

\section{References}

1. Dandona R, Kumar GA, Ameer MA, Ahmed GM, Dandona L. Incidence and burden of road traffic injuries in urban India. Inj Prev. 2008;14(6):354359.

2. National Commission on Macroeconomics and Health, Ministry of Health \& Family Welfare. Burden of disease in India. Injuries in India: A national perspective. 2005 sept;325-350.

3. Pomeranz S, Constantini S, Rappaport ZH. Post operative and intensive care management.in:James E Cottrell,Piyush Patel,David S Warner. Cottrell 
and Patel's Neuroanesthesia: $6^{\text {th }}$ ed.united states of America:Elsevier:2017;66-67.

4. Gaab M, Knoblich OE, Schupp J, Herrmann F, Fuhrmeister U, et al. Effect of furosemide on acute severe experimental cerebral edema. J Neurol.1979 May;220(3):185-197.

5. Askar A, Tarif N.cerebral salt wasting in a patient with head trauma: management with saline hydration and fludrocortisone. Saudi J Kidney Dis Transpl. 2007 march;18(1):95-99.

6. Gribkov AV, Fraerman AP, Salalykin VI, Salmin AA, Sidorkin et al. Regulation of the waterelectrolyte balance during neurosurgical operations with balanced anesthesia using sodium oxybutyrate. AnesteziolReanimatol. 1992;(1):28-31.

7. Rhoney DH, Parker D.Considerations in fluids and electrolytes after traumatic brain injury. NutrClinPract . 2006 oct;21(5):462- 478.

8. Unterberg A, Schneider GH, Gottschalk J, Lanksch WR.Development of traumatic brain edema in old versus young rats. ActaNeurochirSuppl. 1994 jan, 60:431-433.

9. Ishizaki $\mathrm{T}$, Momota $\mathrm{H}$, Kuwahara $\mathrm{K}$, Tanooka A, Morimoto S. A case of symptomatic traumatic cerebral vasospasm associated with hyponatremia. No ShinkeiGeka. 1999; 27(11):1031-1036.

10. James HE, Schneider S. Effects of acute isotonic saline administration on serum osmolality,serum electrolytes, brain water content and intracranial pressure. Acta Neurochir Suppl(Wien). 1993;57:8993.

11. Unterberg A, Kiening K, Schmiedek P, Lanksch W . Long-term observations of intracranial pressure after severe head injury. The phenomenon of secondary rise of intracranial pressure. Neurosurgery. 1993;32(1):17-23.

12. Myshkin KI, Chuenkov VF . Changes in blood serum calcium levels in acute cranio-cerebral injury. Vopr Neirokhir. 1963;27:26-28.

13. Kogan OG, Kaishibaev SK. A case of tetany developing after brain injury. ZdravookhranenieKazakhstana. 1961;21[10]:6870.

14. Gurkoff GG, Shahlaie K, Lyeth BG. In vitro mechanical strain trauma alters neuronal calcium responses: Implications for posttraumatic epilepsy. Epilepsia. 2012 june;53(Suppl 1):53-60.

15. Weisinger JR, Bellorín FE. Magnesium and phosphorus. Lancet. 1992;352(9125):391-396.

16. Audibert G, Hoche J, Baumann A, Mertes PM. Water and electrolytes disorders after brain injury: mechanism and treatment. Ann Fr Anesth Reanim. 2012;31(6):e109-e115.

17. Suman S, Kumar N, Singh Y, Kumar V, Yadav G, et al. Evaluation of Serum Electrolytes in Traumatic Brain Injury Patients: Prospective Randomized Observational Study. J Anesth Crit Care Open Access. 2016;5(3):00184.

18. Mirza Faisal Ahmed Rafiq, Noor Ahmed, Adil Aziz Khan. Serum electrolyte derangements in patients with Traumatic brain injury. J Ayub Med Coll Abbottabad. 2013;25(1-2).

19. Elhassan et al. Association between serum sodium level and outcome of traumatic brain injury among Sudanese patients. Sudan Med J. 2018; 54(3):176181.

20. Gupta SK, Ahuja J, Sharma A. Electrolytes imbalance in traumatic brain injury patients. Medical Education (ASME). 2014 Jan;11:49.

21. Jain A, Gouda B, Gajjar R, Gupta. Correlation Between the Severity of Head Injury And Electrolytes in Patients with Traumatic brain Injury. Journal of Dental and Medical Sciences. 2017; 16(9):55-58.

22. Adiga US, Vickneshwaran V, Sen SK. Electrolyte derangements in traumatic brain injury. Basic Research Journal of Medicine and Clinical Sciences. 2012; 1(2):15-8.

23. Naghibi T, Mohajeri M, Dobakhti F. Inflammation and Outcome in Traumatic Brain Injury: Does Gender Effect on Survival and Prognosis? Journal of clinical and diagnostic research: JCDR. 2017 Feb;11(2):06.

24. Zolin SJ, Vodovotz Y, Forsythe RM, Rosengart MR, Namas R, Brown JB, et al. The early evolving sex hormone environment is associated with significant outcome and inflammatory response differences after injury. Journal of Trauma and Acute Care Surgery. 2015; 78(3):451-58. 